\title{
Thiophosphopeptides Instantly Targeting Golgi Apparatus and Selec- tively Killing Cancer Cells
}

\author{
Weiyi Tan, Qiuxin Zhang, Jiaqing Wang, Meihui Yi, Hongjian He, Bing Xu* \\ Department of Chemistry, Brandeis University, 415 South Street, Waltham, MA 02453, USA
}

Supporting Information Placeholder

ABSTRACT: Golgi apparatus is emerging as a key signaling hub of cells, but there are few approaches for targeting Golgi and selec-
tively killing cancer cells. Here we show an unexpected result that changing an oxygen atom of the phosphoester bond in phospho-
peptides by a sulfur atom enables instantly targeting Golgi apparatus (GA) and selectively killing cancer cells by enzymatic self-
assembly. Specifically, conjugating cysteamine S-phosphate to the C-terminal of a self-assembling peptide generates a thiophospho-
peptide. Being a substrate of alkaline phosphatase (ALP), the thiophosphopeptide undergoes rapid ALP-catalyzed dephosphoryla-
tion to form a thiopeptide that self-assembles. The thiophosphopeptide enters cells via caveolin-mediated endocytosis and
macropinocytosis and instantly accumulates in GA because of dephosphorylation and formation of disulfide bonds in Golgi. Moreo-
ver, the thiophosphopeptide, targeting Golgi, potently and selectively inhibits cancer cells (e.g., HeLa) with the IC 50 (about $3 \mu \mathrm{M}$ ),
which is an order of magnitude more potent than that of the parent phosphopeptide. This work, as the first report of thiophospho-
peptide for targeting Golgi, illustrates a new molecular platform for designing enzyme responsive molecules that target subcellular
compartment for functions.

Golgi apparatus (GA), a stack of flattened membrane-enclosed disks that are dynamically regulated during cell cycles in mammalian cells, is considered as the "heart" of intracellular transportation.1-2 Increasing numbers of studies have revealed that Golgi is a hub for different signaling pathways that drive the survival and migration of cancer cells. ${ }^{3-5}$ Although Golgi is emerging as an important target for cancer therapy, there are, however, few approaches for targeting Golgi.6-7 While Golgi mannosidase II inhibitors are able to inhibit cancer cells, the selectivity $^{8}$ or efficacy ${ }^{6}$ of the inhibitors remains to be improved. In addition, several studies reported the imaging of Golgi, including the commercial dyes for staining Golgi, ${ }^{9}$ a smart "off-on" fluorescence probe for imaging the Golgi in cancer cells, 10 and carbon quantum dots localizing at Golgi. ${ }^{11}$ These imaging agents, however, require 30 minutes $^{10}$ or longer incubation time ${ }^{10}$ or pretreatment, ${ }^{9}$ and they have yet to lead to the approach for selectively inhibiting the cancer cells. Thus, there is an unmet need of targeting Golgi to inhibit cancer cells.

During our study of enzymatic noncovalent synthesis (ENS), ${ }^{12}$ we changed an oxygen atom of the phosphoester bond in a phosphopeptide (p01) by a sulfur atom to make $\mathbf{p S 1}$ for fast enzymatic self-assembly. Our studies show that pS1 undergoes rapid dephosphorylation catalyzed by ALP to form S1 that assembles. Unexpectedly, treating HeLa cells with pS1 shows that $\mathbf{S 1}$ instantly accumulates at Golgi of the HeLa cells at the concentration as low as $500 \mathrm{nM}$. Such an enzymatic accumulation of Golgi (Scheme 1) is proportional to both the concentration of pS1 and the time of incubation. Similar rapid enzymatic accumulation also takes places in the Golgi of several other cells (e.g., Saos2, SJSA1, OVSAHO, HCC1937, and HEK293). Unlike pS1, the parent phosphopeptide, p01, taking longer time for dephosphorylation than that of $\mathbf{p S 1}$, requires hours for cellular uptake and largely remains in endosomes. These results indicate that rapid dephosphorylation of the thiophosphate group and the resulting thiol group are critical for instantly targeting
Golgi. Based on this insight, we designed pS2, a nonfluorescent analogue of pS1. Being able to undergo rapid dephosphorylation catalyzed by ALP to form $\mathbf{S} 2$ that exhibit a critical micelle concentration (CMC) of $9.5 \mu \mathrm{M}$, pS2 inhibits HeLa cells with an $\mathrm{IC}_{50}$ value about $3 \mu \mathrm{M}$, an order of magnitude more potent than that of the parent phosphopeptide. Preliminary mechanistic studies indicate that (i) the thiophosphopeptides enter cells via both caveolin-mediated endocytosis and macropinocytosis, (ii) disulfide bond formation is essential for Golgi targeting, and (iii) the level of ALP of cells contributes to the rate of the accumulation of the resulting thiopeptide assemblies at the Golgi. Providing the first case of targeting Golgi based on fast enzymatic kinetics and oxidative environment of Golgi, this work illustrates a new molecular platform for designing enzyme responsive molecules that target subcellular compartment for functions.

Scheme 1. Illustration of thiophosphopeptides instantly targeting the Golgi apparatus.

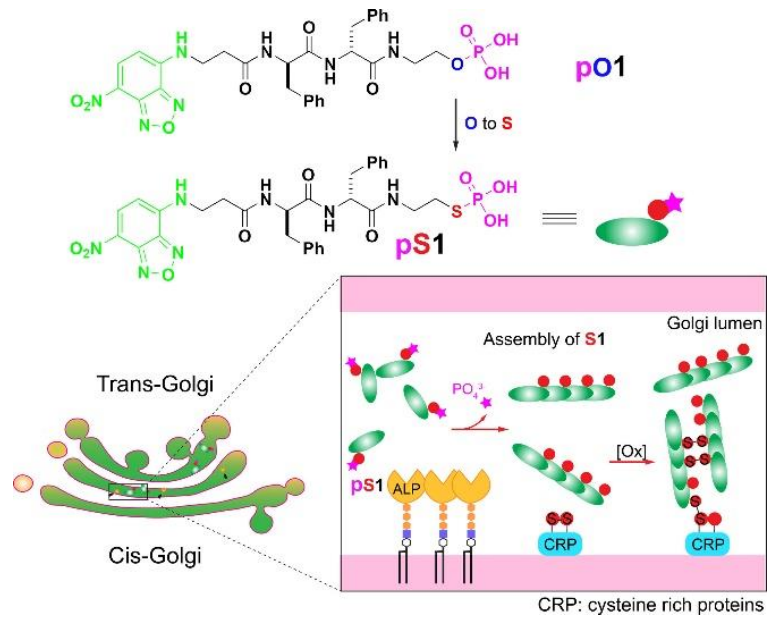


As shown in Scheme 1, pS1 consists of three segments: (i) 4nitro-2,1,3-benzoxadiazole (NBD), a fluorophore that emits bright green fluorescence in hydrophobic environment of supramolecular assemblies; ${ }^{13}$ (ii) D-diphenylalanine (ff), a hydrophobic building block, which enables self-assembly and resists proteolysis; (iii) thiophosphate group, a substrate of ALP14 for enzymatic self-assembly. ${ }^{15-16}$ Solid phase peptide synthesis (SPPS) of NBD-ff followed by a conjugation of cysteamine Sphosphate ${ }^{17}$ generates pS1 (Scheme S2) in a good yield. Such a design ensures the fast dephosphorylation of the thiophosphopeptide by ALP (Figure S1). While pS1 exhibits critical micelle concentration (CMC) of $6.0 \mu \mathrm{M}, \mathbf{S 1}$ has the CMC of $2.4 \mu \mathrm{M}$ (Figure S2). Transmission electron microscopy (TEM) reveals that pS1, at $5 \mu \mathrm{M}$ form micelles, which turn into nanofibers after ALP converts pS1 to $\mathbf{S 1}$ (Figure S3). The formation of micelles of pS1 likely facilitates the cellular uptake by caveolin-mediated endocytosis, similar to the cellular uptake of peptide amphiphiles. ${ }^{18-21}$

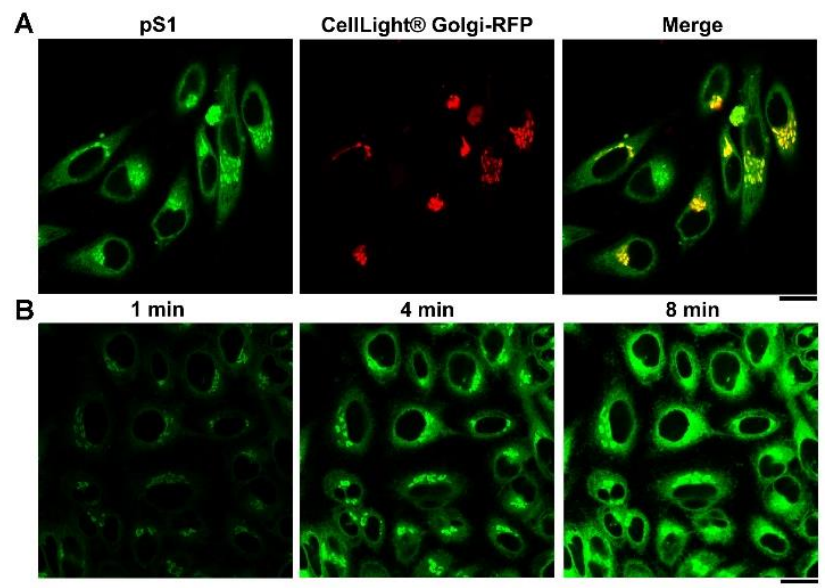

Figure 1. CLSM images of HeLa cells (A) stained with CellLight $\AA$ Golgi-RFP after treating with pS1 for 8 minutes and (B) treated with pS1 for 1, 4, and $8 \mathrm{~min}$. Scale bars $=20 \mu \mathrm{m}$ and $[\mathbf{p S 1}]=10 \mu \mathrm{M}$.

We first incubated HeLa cells with CellLight ${ }^{\circledR}$ Golgi-RFP22 for 24 hours to transfect RFP at the Golgi, then incubated the HeLa cells with pS1 $(10 \mu \mathrm{M})$ for 8 minutes (Figure 1$)$. The fluorescence from the assemblies of $\mathbf{S 1}$ overlaps with the red fluorescence from all Golgi-RFP, confirming that pS1 targets the Golgi of the HeLa cells. The fluorescence of $\mathbf{S 1}$ appears almost instantly after adding $\mathbf{p S 1}$ in the culture of HeLa cells (Figure S4). This rate is, at least an order of magnitude, faster than previously reported probes. ${ }^{9-11}$ The intensity of the fluorescence at the Golgi increases significantly with the time of incubation of pS1, about 7 times enhancement from 1 minute to 8 minutes. Except the bright fluorescence at the Golgi and the dim fluorescence at the endoplasmic reticulum (ER), the rest of intracellular and extracellular regions of the HeLa remain dark. This result indicates that enzymatic assembly of $\mathbf{S 1}$ occurs at the Golgi of HeLa cells, agreeing with the observation of ALP at Golgi of HeLa cells. ${ }^{23}$ Live cell imaging over 20 minutes (Video S1) reveals that the fluorescence of the assemblies of $\mathbf{S 1}$ emerges at the Golgi prior to diffusing to ER, likely resulted from Golgi-ER transport. ${ }^{2}$ The differential interference contrast (DIC) image of HeLa cells treated with $10 \mu \mathrm{M}$ of $\mathbf{p S 1}$ for 20 min (Figure S5) shows well-spread HeLa cells, excluding that pS1 enters cells due to cell death.

The concentration of $\mathbf{p S 1}$ is another important factor determines the accumulation of $\mathbf{S 1}$ at Golgi. We compared the Golgi fluorescence by fixing the incubation time at 4 minutes and varying the concentration $\mathbf{p S 1}$ at 10,5 , and $2 \mu \mathrm{M}$ (Figure 2). At 10 $\mu \mathrm{M}$, bright green fluorescence presents in Golgi and weak fluorescence in ER; at $5 \mu \mathrm{M}$, green fluorescence clearly still presents at the Golgi, but little at the ER; at $2 \mu \mathrm{M}$, much weaker fluorescence at the Golgi. Further decreasing the concentration of pS1 to $500 \mathrm{nM}$ still results in Golgi accumulation of S1 in HeLa cells. Though the brightness of $\mathbf{S 1}$ at Golgi is weaker at the beginning of the addition, distinctive fluorescence appears at the Golgi after 15 minutes (Figure S6). The accumulation rate of $\mathbf{S 1}$ at Golgi, quantified by the increase of the fluorescence intensity, is concentration dependent (Figure S7). Using a Golgi disruptor, brefeldin A (BFA), is able to abolish the accumulation of S1 (Figure S8). These results indicate that enzymatic formation and self-assembly of $\mathbf{S 1}$ in situ at Golgi enables the instant targeting of Golgi. In addition, the concentration needed of pS1 for targeting Golgi is much less than the previously reported probes. $^{9-11}$
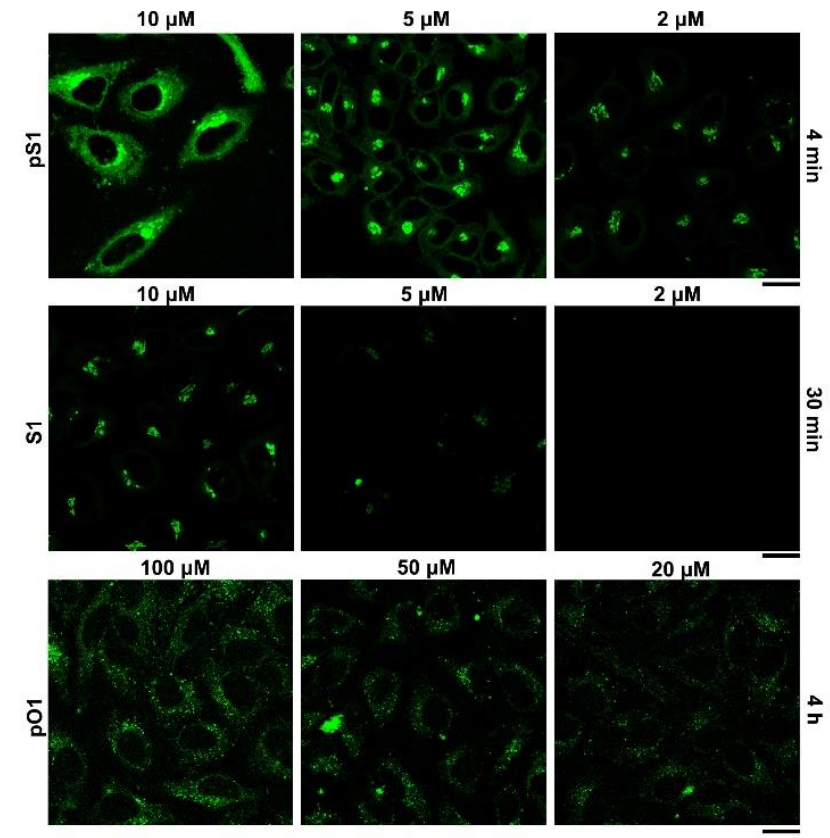

Figure 2. CLSM images of HeLa cells treated with pS1 $(10 \mu \mathrm{M}$, $5 \mu \mathrm{M}, 2 \mu \mathrm{M})$ for $4 \min ($ top), $\mathbf{S} \mathbf{1}(10 \mu \mathrm{M}, 5 \mu \mathrm{M}, 2 \mu \mathrm{M})$ for $30 \mathrm{~min}$ (middle) and p01 $(100 \mu \mathrm{M}, 50 \mu \mathrm{M}, 20 \mu \mathrm{M})$ for $4 \mathrm{~h}$. Scale bars = $20 \mu \mathrm{m}$.

Unlike pS1, S1, at the concentration of $10 \mu \mathrm{M}$ and being incubated with HeLa cells for 8 minutes, hardly results in any fluorescence in the cells (Figure S9A), suggesting slower cell uptake of $\mathbf{S 1}$ than that of $\mathbf{p S 1}$ and indicating the importance of enzymatic dephosphorylation for targeting Golgi. As shown in Figure 2, after incubating $\mathbf{S 1}(10 \mu \mathrm{M})$ with HeLa cells for 30 minutes, some green fluorescence at the Golgi of the HeLa cells, with the fluorescent intensity similar to that of HeLa cells incubated with pS1 $(2 \mu \mathrm{M})$ for 4 minutes. After 30 minutes incubation, when the concentrations of $\mathbf{S 1}$ are at 5 and $2 \mu \mathrm{M}$, there is very weak at the Golgi and no fluorescence in cell at all, respectively. These results suggest that $\mathbf{S 1}$ enters the HeLa cells less efficiently than pS1 does. Moreover, p01 (the parent compound of pS1) produces almost no fluorescence (Figure S9B) in the HeLa cells after 8 minutes incubation and at the concentration of $10 \mu \mathrm{M}$. In fact, after 4 hours of incubation of p01 and HeLa cells, there are several scattered fluorescent puncta in cells, with fluorescent intensity being proportional to the concentrations of p01 (from 100 to 50 and to $20 \mu \mathrm{M}$ ). These 
results indicate that the assemblies of $\mathbf{0 1}$, formed by dephosphorylation, largely are retained in endosomes or lysosomes (Figure 2, Scheme S3). The above results confirm the unique ability of $\mathbf{p S 1}$ for instantly targeting Golgi.

A

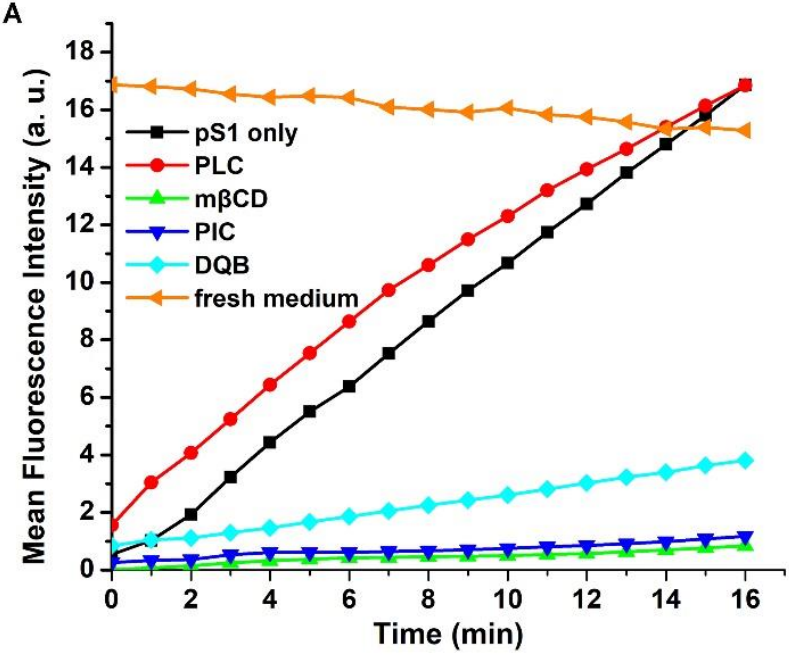

B

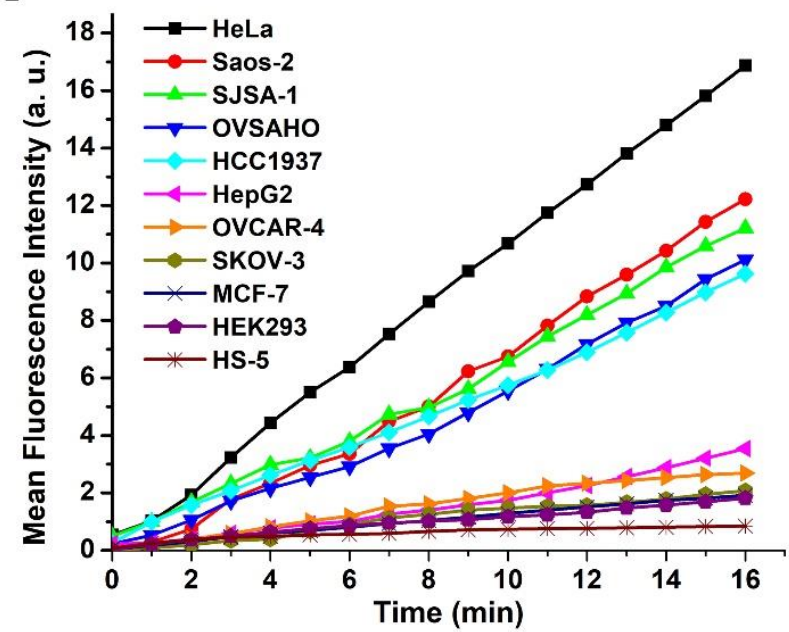

Figure 3. Time-dependent mean fluorescence intensity of Golgi in (A) HeLa cells pretreated with PLC (phospholipase C, $0.2 \mathrm{U}$, $30 \mathrm{~min}$ ), $\mathrm{m} \beta C D(5 \mathrm{mM}, 30 \mathrm{~min}$ ), PIC (phosphatase inhibitor cocktail set $3,4000 \times, 30 \mathrm{~min})$, DQB $(20 \mu \mathrm{M}, 30 \mathrm{~min})$, respectively, and then treated with pS1 $(10 \mu \mathrm{M})$, and the pS1 treated HeLa cells in a fresh medium. (B) Time-dependent mean fluorescence intensity of Golgi in different cell lines treated with pS1 $(10 \mu \mathrm{M})$.

To further understand the mechanism of Golgi-accumulation of $\mathbf{S 1}$ assemblies resulted from the rapid enzymatic dephosphorylation of $\mathbf{p S 1}$, we examined the rate of fluorescence increase in Golgi for 16 minutes in the HeLa cells treated pS1 and several inhibitors, using the fluorescence increase in the Golgi of the HeLa cells incubated with pS1 only as the reference (Figure 3A). Using phospholipase C (PLC), ${ }^{24}$ an enzyme that cleaves glycosylphosphatidylinositol (GPI) anchor, to remove ALP from the cell membrane results in slightly faster increase of fluorescence in the Golgi, confirming that ALP at Golgi dephosphorylates pS1 and indicating that pericellular dephosphorylation of pS1 by the ALP on plasma membrane slightly slows down the accumulation of S1 at the Golgi. Methyl$\beta$-cyclodextrin $(m \beta C D)$ significantly decreases the rate of the fluorescence increase at the Golgi, indicating that pS1 (at $10 \mu \mathrm{M}$ ) likely enters cells via caveolin-mediated endocytosis. As a potent inhibitor of actin polymerization, ${ }^{25}$ cytochalasin D (CytD) decreases the accumulation of $\mathbf{S 1}$ at Golgi in a concentrationdependent manner (Figure S10). This result agrees with the critical role of actin dynamics in cellular uptake, indicating that pS1 also enters the cells via marcopinocytosis. ${ }^{26}$ Both the phosphatase inhibitor cocktail set 3 (PIC) and the tissue nonspecific ALP inhibitor $\left(\mathrm{DQB}^{27}\right)$ reduce the rate of the fluorescence increase at the Golgi, with PIC more effectively inhibiting the accumulation than DQB. These results suggest that other phosphatases, in addition to ALP, also contribute to the enzymatic accumulation of $\mathbf{S 1}$ at Golgi and agree with sorting of ALP at the Golgi before secretion. ${ }^{28-29}$

We also examined the exocytosis of the accumulated $\mathbf{S 1}$ by incubating the HeLa cells pretreated with pS1 in a fresh culture medium. The intensity of fluorescence at the Golgi of the HeLa cells drops only slightly over time, confirming that the enzymatically formed assemblies of $\mathbf{S 1}$ are largely trapped in the Golgi. Inhibiting protein disulfide isomerases (PDIs) decrease disulfide bonds of cysteine rich proteins (CRPs) ${ }^{30}$ that are transported to Golgi, contributing to a slight decrease of the rate of Golgi accumulation of S1 (Figure S11). This result implies the peptide assemblies likely form disulfide bonds with CRPs. This observation agrees with that dimers of S1 (or S2) form in the cell lysate treated with pS1 (or pS2) (Figure S12), suggesting that certain extent of covalent linkage between assemblies likely contributes the retention of the assemblies in the Golgi. Moreover, the addition of N-ethylmaleimide almost completely eliminates the accumulation of $\mathbf{S 1}$ at Golgi (Figure S13), further supporting that $\mathbf{S 1}$ forms disulfide bond with CRPs at Golgi.

To examine the applicability of the process illustrated in Scheme 1 for targeting Golgi of other cells, we incubated pS1 with several other cancer cell lines (Saos-2, SJSA-1, OVSAHO, HCC1937, HepG2, OVCAR-4, SKOV-3, MCF-7) and immortalized normal cell lines (HEK293 and HS-5) and examined the rates of fluorescent increase at the Golgi of the cells (Figure 3B). The fluorescence intensities increase significantly at the Golgi of Saos-2, SJSA-1, OVSAHO and HCC1937 cells, slightly in those of HepG2 and OVCAR4 cells, and much slowly in those of SKOV3, MCF7, HEK293 and HS-5 cells. These results largely agree with expression levels of ALP in these cell (Figure S14). ${ }^{31}$ One exception is HepG2, which expresses higher level of ALP than OVSAHO, but exhibits slower fluorescence increase at Golgi. High level of glutathione in hepatocytes ${ }^{32}$ likely antagonizes the accumulation of $\mathbf{S 1}$ in the Golgi. This observation supports that oxidative Golgi environments favors disulfide formation, thus the retention of the assemblies of $\mathbf{S 1}$ at the Golgi.

We synthesized a nonfluorescent analogue (pS2) of pS1 by using naphthyl group to replace NBD (Figure S15). Being similar as pS1, pS2 undergoes rapid dephosphorylation by ALP to form S2 (Figure S16). Compared with its oxophosphate analogue p02,33 pS2 shows much faster dephosphorylation. For example, being incubated with ALP $(0.1 \mathrm{U} / \mathrm{mL})$ for about 16 minutes, pS2 nearly fully converted to $\mathbf{S 2}$, while the maximum conversion ratio of $\mathbf{p 0 2}$ to $\mathbf{0 2}$ remains at about $50 \%$ at the same duration (Figure S16). The CMC of pS2 is $9.5 \mu \mathrm{M}$, and the CMC of its dephosphorylated product, S2, is $4.3 \mu \mathrm{M}$ (Figure S17), indicating both compounds have an excellent self-assembling ability. We further tested the cytotoxicity of pS2 against HeLa, HEK293, and HS-5 cells and found the IC50 values are 2.8 $\mu \mathrm{M},>100 \mu \mathrm{M}$ and $>100 \mu \mathrm{M}$, respectively (Figure $4 \mathrm{~A}$ ). The inhibitory activity of $\mathbf{p S 2}$ against HeLa cells is an order of magnitude higher than that of p02 (Figure S18). The difference between these $\mathrm{IC}_{50}$ values agrees with the difference of the rate of enzymatic assemblies in Golgi of the cells, indicating that selectively targeting the Golgi is resulted from fast enzyme kinetics. This 
bioRxiv preprint doi: https://doi.org/10.1101/2021.02.13.431079; this version posted February 14, 2021. The copyright holder for this preprint (which was not certified by peer review) is the author/funder. All rights reserved. No reuse allowed without permission.

result also confirms that $\mathbf{p S 2}$ is more selective than $\mathbf{S 2}$ against cancer cells (Figure S19). In addition, several commonly used inhibitors (Z-VAD-FMK, NAc, Nec-1, DFO, Fer-1, and disulfiram $)^{34}$ of cell death are unable to rescuing HeLa cells from $\mathbf{p S 2}$ (Figures S20 and S21), suggesting a unique cell death resulted from the molecular processes defined thiophosphopeptides at the Golgi apparatus. Treating the HeLa cells incubated with pS2

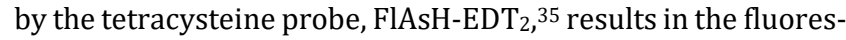
cent at the Golgi of the HeLa cells (shown by the arrow in Figures 4B and S22), indicating that S2 self-assembles at the Golgi to arrange multiple $\mathrm{C}$-terminal thiols into arrangement similar to that of tetracysteine.

A
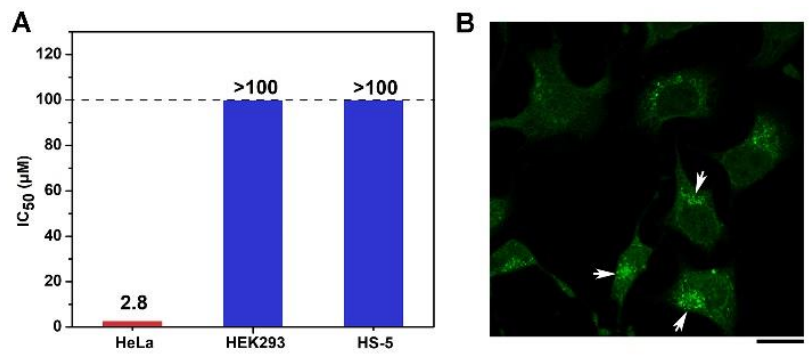

Figure 4. (A) IC 50 of pS2 against HeLa cells, HEK293 cells and HS-5 cells. (B) CLSM images of HeLa cells treated with pS2 (10 $\mu \mathrm{M}, 4 \mathrm{~h}$ ) and then stained by a tetracysteine probe, FlAsH-EDT2. Scale bar $=20 \mu \mathrm{m}$.

In summary, this work illustrates that rapid dephosphorylation of thiophosphopeptides enables instantly targeting of Golgi apparatus and selectively inhibiting the cancer cells. Our observations agree with several known facts: (i) CRPs are enriched in Golgi,36-38 (ii) a significant level of oxidation occurs in the Golgi membrane, ${ }^{39}$ (iii) ALP, as an "almost perfect" enzyme $^{40}$ being anchored on the cell membrane by glycosylphosphatidylinositol (GPI) and overexpressed on certain cancer cell, ${ }^{23}, 41-42$ is known to be sorted as oligomers at the Golgi before secretion. ${ }^{28-29}$ Since thiophosphopeptides ${ }^{43-45}$ are much less developed than phosphopeptides, replace NBD with other functional motifs (e.g. 10-hydroxycamptothecine) ${ }^{46}$ may lead to new discoveries. These thiophosphopeptides also may serve as the substrates for thiol-click chemistry 47 or for integration thiol groups in other supramolecular assemblies. ${ }^{48-52}$

\section{ASSOCIATED CONTENT}

\section{AUTHOR INFORMATION}

\section{Corresponding Author}

Bing Xu - Department of Chemistry, Brandeis University, 415 South Street, Waltham, MA 02453, USA; Email: bxu@brandeis.edu

\section{Authors}

Weiyi Tan - Department of Chemistry, Brandeis University, 415 South Street, Waltham, MA 02453, USA

Qiuxin Zhang - Department of Chemistry, Brandeis University, 415 South Street, Waltham, MA 02453, USA

Jiaqing Wang - Department of Chemistry, Brandeis University, 415 South Street, Waltham, MA 02453, USA
Meihui Yi - Department of Chemistry, Brandeis University, 415 South Street, Waltham, MA 02453, USA

Hongjian He - Department of Chemistry, Brandeis University, 415 South Street, Waltham, MA 02453, USA

Notes

The authors declare no competing financial interests.

\section{ACKNOWLEDGMENT}

This work is partially supported by NIH (CA142746, CA252364) and NSF (DMR-2011846).

\section{REFERENCES}

1. Kulkarni-Gosavi, P.; Makhoul, C.; Gleeson, P. A., Form and function of the Golgi apparatus: scaffolds, cytoskeleton and signalling. FEBS. Lett. 2019, 593, 2289-2305.

2. Lee, M. C.; Miller, E. A.; Goldberg, J.; Orci, L.; Schekman, R., Bidirectional protein transport between the ER and Golgi. Annu. Rev. Cell. Dev. Biol. 2004, 20, 87-123.

3. Bivona, T. G.; Pérez de Castro, I.; Ahearn, I. M.; Grana, T. M.; Chiu, V. K.; Lockyer, P. J.; Cullen, P. J.; Pellicer, A.; Cox, A. D.; Philips, M. R., Phospholipase $\mathrm{C} \gamma$ activates Ras on the Golgi apparatus by means of RasGRP1. Nature 2003, 424, 694-698.

4. Farber-Katz, S. E.; Dippold, H. C.; Buschman, M. D.; Peterman, M. C.; Xing, M.; Noakes, C. J.; Tat, J.; Ng, M. M.; Rahajeng, J.; Cowan, D. M.; Fuchs, G. J.; Zhou, H.; Field, S. J., DNA damage triggers golgi dispersal via DNA-PK and GOLPH3. Cell 2014, 156, 413-427.

5. Rieger, L.; O’Shea, S.; Godsmark, G.; Stanicka, J.; Kelly, G.; O’Connor, R., IGF-1 receptor activity in the Golgi of migratory cancer cells depends on adhesion-dependent phosphorylation of Tyr1250 and Tyr1251. Sci. Signal. 2020, 13 .

6. Armstrong, Z.; Kuo, C. L.; Lahav, D.; Liu, B.; Johnson, R.; Beenakker, T. J. M.; De Boer, C.; Wong, C. S.; Van Rijssel, E. R.; Debets, M. F.; Florea, B. I.; Hissink, C.; Boot, R. G.; Geurink, P. P.; Ovaa, H.; Van Der Stelt, M.; Van Der Marel, G. M.; Codée, J. D. C.; Aerts, J. M. F. G.; Wu, L.; Overkleeft, H. S.; Davies, G. J., Manno- epi-cyclophellitols Enable Activity-Based Protein Profiling of Human $\alpha$-Mannosidases and Discovery of New Golgi Mannosidase II Inhibitors. J. Am. Chem. Soc. 2020, 142, 13021-13029.

7. Van Den Elsen, J. M. H.; Kuntz, D. A.; Rose, D. R., Structure of Golgi $\alpha$-mannosidase II: A target for inhibition of growth and metastasis of cancer cells. Embo. J. 2001, 20, 3008-3017.

8. Dennis, J. W.; Koch, K.; Yousefi, S.; VanderElst, I., Growth inhibition of human melanoma tumor xenografts in athymic nude mice by swainsonine. Cancer Res. 1990, 50, 1867-72.

9. van Echten-Deckert, G.; Giannis, A.; Schwarz, A.; Futerman, A. H.; Sandhoff, K., 1-Methylthiodihydroceramide, a novel analog of dihydroceramide, stimulates sphinganine degradation resulting in decreased de novo sphingolipid biosynthesis. J. Biol. Chem. 1998, 273, 1184-91.

10. Zhang, H.; Fan, J.; Wang, J.; Zhang, S.; Dou, B.; Peng, X., An off-on COX-2-specific fluorescent probe: Targeting the golgi apparatus of cancer cells. J. Am. Chem. Soc. 2013, 135, 11663-11669.

11. Li, R. S.; Gao, P. F.; Zhang, H. Z.; Zheng, L. L.; Li, C. M.; Wang, J.; Li, Y. F.; Liu, F.; Li, N.; Huang, C. Z., Chiral nanoprobes for targeting and long-term imaging of the Golgi apparatus. Chem. Sci. 2017, 8, 6829-6835. 12. He, H.; Tan, W.; Guo, J.; Yi, M.; Shy, A. N.; Xu, B., Enzymatic Noncovalent Synthesis. Chem. Rev. 2020, 120, 9994-10078.

13. Gao, Y.; Shi, J.; Yuan, D.; Xu, B., Imaging enzyme-triggered selfassembly of small molecules inside live cells. Nat. Commun. 2012, 3, 1-8. 14. Herrington, K. A.; Small, C. J.; Meister, A.; Friedman, O. M., Studies on Latent Derivatives of Aminoethanethiols as Potentially Selective Cytoprotectants IV. Enzymatic Hydrolysis of Cysteamine-S-phosphate. Cancer Res. 1967, 27, 148-151.

15. Yang, Z.; Gu, H.; Fu, D.; Gao, P.; Lam, J. K.; Xu, B., Enzymatic formation of supramolecular hydrogels. Adv. Mater. 2004, 16, 1440-1444. 16. Zhou, J.; Du, X.; Li, J.; Yamagata, N.; Xu, B., Taurine boosts cellular uptake of small D-peptides for enzyme-instructed intracellular molecular self-assembly. J. Am. Chem. Soc. 2015, 137, 10040-10043. 
17. Åkerfeldt, S., Hydrolysis of Cysteamine S-Phosphate. J. Org. Chem. 1964, 29, 493.

18. Liang, C.; Yan, X.; Zhang, R.; Xu, T.; Zheng, D.; Tan, Z.; Chen, Y.; Gao, Z.; Wang, L.; Li, X.; Yang, Z., Enhanced cellular uptake and nuclear accumulation of drug-peptide nanomedicines prepared by enzymeinstructed self-assembly. J. Control. Release. 2020, 317, 109-117.

19. Lock, L. L.; Reyes, C. D.; Zhang, P.; Cui, H., Tuning Cellular Uptake of Molecular Probes by Rational Design of Their Assembly into Supramolecular Nanoprobes. J. Am. Chem. Soc. 2016, 138, 3533-3540.

20. Wang, Y.; Li, Z.; Shmidov, Y.; Carrazzone, R. J.; Bitton, R.; Matson, J. B., Crescent-Shaped Supramolecular Tetrapeptide Nanostructures. J. Am. Chem. Soc. 2020, 142, 20058-20065.

21. Feng, Z.; Wang, H.; Wang, S.; Zhang, Q.; Zhang, X.; Rodal, A. A.; Xu, B., Enzymatic Assemblies Disrupt the Membrane and Target Endoplasmic Reticulum for Selective Cancer Cell Death. J. Am. Chem. Soc. 2018, 140, 9566-9573.

22. Herrera, C.; Klokk, T. I.; Cole, R.; Sandvig, K.; Mantis, N. J., A bispecific antibody promotes aggregation of ricin toxin on cell surfaces and alters dynamics of toxin internalization and trafficking. PLoS One 2016, 11. 23. Sasaki, M.; Fishman, W. H., Ultrastructural Studies on Regan and NonRegan Isoenzymes of Alkaline Phosphatase in Human Ovarian Cancer Cells. Cancer Res. 1973, 33, 3008-3018.

24. Low, M. G.; Finean, J. B., Release of alkaline phosphatase from membranes by a phosphatidylinositol-specific phospholipase C. Biochem. J. 1977, 167, 281-284.

25. Gottlieb, T. A.; Ivanov, I. E.; Adesnik, M.; Sabatini, D. D., Actin microfilaments play a critical role in endocytosis at the apical but not the basolateral surface of polarized epithelial cells. J Cell Biol 1993, 120, 695710.

26. Nakase, I.; Niwa, M.; Takeuchi, T.; Sonomura, K.; Kawabata, N.; Koike, Y.; Takehashi, M.; Tanaka, S.; Ueda, K.; Simpson, J. C., Cellular uptake of arginine-rich peptides: roles for macropinocytosis and actin rearrangement. Mol. Ther. 2004, 10, 1011-1022.

27. Dahl, R.; Sergienko, E. A.; Su, Y.; Mostofi, Y. S.; Yang, L.; Simao, A. M.; Narisawa, S.; Brown, B.; Mangravita-Novo, A.; Vicchiarelli, M.; Smith, L. H.; O’Neill, W. C.; Millán, J. L.; Cosford, N. D. P., Discovery and Validation of a Series of Aryl Sulfonamides as Selective Inhibitors of Tissue-Nonspecific Alkaline Phosphatase (TNAP). J. Med. Chem. 2009, 52, 6919-6925.

28. Goldfischer, S., The internal reticular apparatus of Camillo Golgi: a complex, heterogeneous organelle, enriched in acid, neutral, and alkaline phosphatases, and involved in glycosylation, secretion, membrane flow, lysosome formation, and intracellular digestion. J. Histochem. Cytochem 1982, 30, 717-33.

29. Paladino, S.; Lebreton, S.; Tivodar, S.; Formiggini, F.; Ossato, G.; Gratton, E.; Tramier, M.; Coppey-Moisan, M.; Zurzolo, C., Golgi sorting regulates organization and activity of GPI proteins at apical membranes. Nat. Chem. Biol. 2014, 10, 350-357.

30. Lyles, M. M.; Gilbert, H. F., Catalysis of the oxidative folding of ribonuclease $\mathrm{A}$ by protein disulfide isomerase: pre-steady-state kinetics and the utilization of the oxidizing equivalents of the isomerase. Biochemistry 1991, 30, 619-625.

31. Rouillard, A. D.; Gundersen, G. W.; Fernandez, N. F.; Wang, Z.; Monteiro, C. D.; McDermott, M. G.; Ma'ayan, A., The harmonizome: a collection of processed datasets gathered to serve and mine knowledge about genes and proteins. Database 2016, 2016.

32. Kretzschmar, M., Regulation of hepatic glutathione metabolism and its role in hepatotoxicity. Exp. Toxicol. Pathol. 1996, 48, 439-446.

33. Feng, Z.; Wang, H.; Yi, M.; Lo, C.-Y.; Sallee, A.; Hsieh, J.-T.; Xu, B., Instructed-assembly of small peptides inhibits drug-resistant prostate cancer cells. Pept. Sci. 2020, 112, e24123.

34. Tang, D.; Kang, R.; Berghe, T. V.; Vandenabeele, P.; Kroemer, G., The molecular machinery of regulated cell death. Cell Res. 2019, 29, 347-364.
35. Adams, S. R.; Campbell, R. E.; Gross, L. A.; Martin, B. R.; Walkup, G. K.; Yao, Y.; Llopis, J.; Tsien, R. Y., New Biarsenical Ligands and Tetracysteine Motifs for Protein Labeling in Vitro and in Vivo: Synthesis and Biological Applications. J. Am. Chem. Soc. 2002, 124, 6063-6076. 36. Aoki, D.; Lee, N.; Yamaguchi, N.; Dubois, C.; Fukuda, M. N., Golgi retention of a trans-Golgi membrane protein, galactosyltransferase, requires cysteine and histidine residues within the membrane-anchoring domain. Proc. Natl. Acad. Sci. U. S. A. 1992, 89, 4319-4323.

37. Maeda, Y.; Beznoussenko, G. V.; Van Lint, J.; Mironov, A. A.; Malhotra, V., Recruitment of protein kinase D to the trans - Golgi network via the first cysteine - rich domain. EMBO J. 2001, 20, 5982-5990.

38. Zhang, W.; Zhang, J.; Li, P.; Liu, J.; Su, D.; Tang, B., Two-photon fluorescence imaging reveals a Golgi apparatus superoxide anion-mediated hepatic ischaemia-reperfusion signalling pathway. Chem. Sci. 2019, 10, 879-883.

39. Hatori, Y.; Kubo, T.; Sato, Y.; Inouye, S.; Akagi, R.; Seyama, T., Visualization of the redox status of cytosolic glutathione using the organelle-and cytoskeleton-targeted redox sensors. Antioxidants 2020, 9.

40. Simopoulos, T. T.; Jencks, W. P., Alkaline Phosphatase Is an Almost Perfect Enzyme. Biochemistry 1994, 33, 10375-10380.

41. Fishman, W. H.; Inglis, N. R.; Green, S.; Anstiss, C. L.; Gosh, N. K.; Reif, A. E.; Rustigian, R.; Krant, M. J.; Stolbach, L. L., Immunology and biochemistry of Regan isoenzyme of alkaline phosphatase in human cancer. Nature 1968, 219, 697-699.

42. Vijayan, D.; Young, A.; Teng, M. W. L.; Smyth, M. J., Targeting immunosuppressive adenosine in cancer. Nat. Rev. Cancer 2017, 17, 709724.

43. Parker, L. L.; Schilling, A. B.; Kron, S. J.; Kent, S. B. H., Optimizing thiophosphorylation in the presence of competing phosphorylation with MALDI-TOF-MS detection. J. Proteome Res. 2005, 4, 1863-1866.

44. KÜNg, E. K. E.; Bannwarth, W., Chemical synthesis of o thiophosphotyrosyl peptides. Int. J. Pept. Protein Res. 1994, 43, 146-153. 45. Attard, T. J.; O'Brien-Simpson, N.; Reynolds, E. C., Synthesis of phosphopeptides in the Fmoc mode. Int. J. Pept. Res. Ther. 2007, 13, 447468.

46. Cai, Y.; Shen, H.; Zhan, J.; Lin, M.; Dai, L.; Ren, C.; Shi, Y.; Liu, J.; Gao, J.; Yang, Z., Supramolecular "Trojan Horse" for Nuclear Delivery of Dual Anticancer Drugs. J. Am. Chem. Soc. 2017, 139, 2876-2879.

47. Liang, G.; Ren, H.; Rao, J., A biocompatible condensation reaction for controlled assembly of nanostructures in living cells. Nat. Chem. 2010, 2 , 54-60.

48. Shigemitsu, H.; Kubota, R.; Nakamura, K.; Matsuzaki, T.; Minami, S.; Aoyama, T.; Urayama, K.; Hamachi, I., Protein-responsive protein release of supramolecular/polymer hydrogel composite integrating enzyme activation systems. Nat. Commun. 2020, 11, 3859.

49. Tanaka, A.; Fukuoka, Y.; Morimoto, Y.; Honjo, T.; Koda, D.; Goto, M.; Maruyama, T., Cancer Cell Death Induced by the Intracellular SelfAssembly of an Enzyme-Responsive Supramolecular Gelator. J. Am. Chem. Soc. 2015, 137, 770-775.

50. Pires, R. A.; Abul-Haija, Y. M.; Costa, D. S.; Novoa-Carballal, R.; Reis, R. L.; Ulijn, R. V.; Pashkuleva, I., Controlling Cancer Cell Fate Using Localized Biocatalytic Self-Assembly of an Aromatic Carbohydrate Amphiphile. J. Am. Chem. Soc. 2015, 137, 576-579.

51. Merg, A. D.; Touponse, G.; van Genderen, E.; Zuo, X.; Bazrafshan, A.; Blum, T.; Hughes, S.; Salaita, K.; Abrahams, J. P.; Conticello, V. P., 2D Crystal Engineering of Nanosheets Assembled from Helical Peptide Building Blocks. Angew. Chem. Int. Ed. 2019, 58, 13507-13512.

52. Liu, Q.; Yuan, Z.; Guo, X.; van Esch, J. H., Dual-Functionalized Crescent Microgels for Selectively Capturing and Killing Cancer Cells. Angew. Chem. Int. Ed. 2020, 59, 14076-14080. 


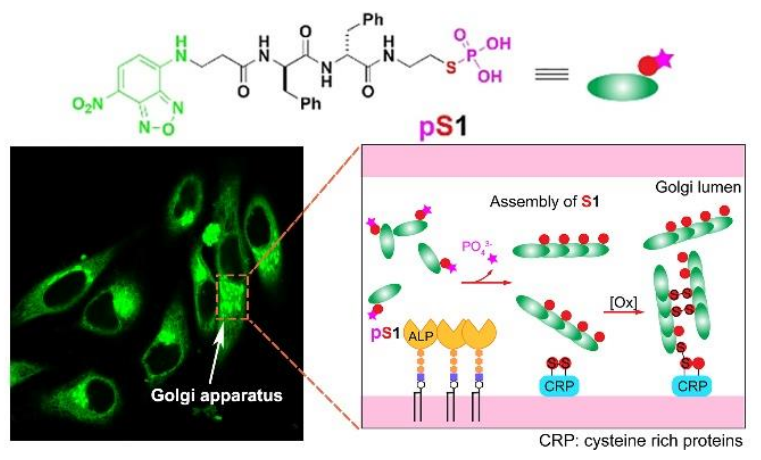

